\title{
DESIGN OF A MAGNETIC OPTICAL SYSTEM FOR TRANSPORT AND MATCHING OF MULTIPLE-CHARGE-STATE HEAVY-ION BEAMS *
}

\author{
M. Portillo, V.N. Aseev, J.A. Nolen, P.N. Ostroumov \\ Physics Division, ANL, 9700 S. Cass Ave., Argonne, IL 60439, USA
}

\begin{abstract}
The requirements of the design of beam transport systems for selecting multiple charge state beams are discussed. The layouts of two designs are presented along with the results calculated from beam transport maps.
\end{abstract}

\section{INTRODUCTION}

A proposal for a high power driver linac intended for the Rare Isotope Accelerator (RIA) requires systems for enhancing beam intensity [1]. A cost effective linac sets a limit on the highest $m / q$ ratio that may be accelerated with maximum energy at minimal losses. Current ion source technology is limited at the $\mathrm{m} / \mathrm{q}$ necessary to deliver high flux beams such of very heavy ions such as lead or uranium. A possible solution is to design an accelerator system that can accept a wide enough fractional range of $\mathrm{m} / \mathrm{q}$ states to satisfy the demand for beam intensities of such beams [2].

An important aspect of such a driver linac system is the transport of the beam after stripping. Most charge stripping systems in accelerators are followed by a magnetic spectrometer system for selecting out a single $\mathrm{m} / \mathrm{q}$ state of interest. Multiple $q$ systems are required here with the following characteristics taken into account:

(1) Capable of transporting a minimum range of $m / q$ states.

(2) Section of high dispersion for selecting off unwanted charge states after stripping.

(3) Section with rebunching to match the longitudinal phase space of the beam to rf structures.

(4) Achromatic character to minimize dispersion in the horizontal plane at the rebuncher and at the exit.

(5) Isopath character to minimize time of flight dispersion and preserve the arrival time at the rf structures for different charge states.

(6) Rotate direction and translate position of the beam to accommodate the housing of the facility.

Two distinct systems with these characteristics are considered in this analysis.

\section{BEAM CHARACTERISTICS}

Before carrying out any transport calculations the phase space characteristics of the beam need to be determined. Starting from the ionization and extraction of the ions, all proceeding sections of the acceleration and transport

*Work supported by the U. S. Department of Energy, Nuclear Physics Division, under Contract W-31-109-ENG-38. contribute to the phase space occupied by the beam. These parameters are determined from past experience with other beams along with estimates of the effects by the acceleration and transport systems intended for the driver linac [1].

A survey was done on the effects of stripper foils on particles for the appropriate energies. Various models [3] and tabulated data [4] are available for this task. The cases for stripping of $238 \mathrm{U}$ required special attention since energy straggling can set limits on the maximum foil thickness allowable for obtaining high mean $q$. The beam parameters will be specified below.

\section{BEAM TRANSPORT CALCULATIONS}

Higher order effects need to be considered for the electromagnetic elements applied in the transport system. This is primarily important since the charge states become dispersed and will experience slightly different fields. Ray tracing calculations are ideal for determining higher order effects; however, the complexity of these systems require mapping methods to arrive at first order solutions.

The COSY Infinity code system is adopted our calculations. It is capable of evaluating maps to arbitrary order and is equipped with efficient fitting algorithms [5][6]. A version of the code was modified to calculate maps of elements with time-varying electromagnetic fields [7]. This was necessary for simulating the effects of the rf cavities used for rebunching.

The transfer maps were carried out to 3rd order to determine the effects on the phase space of each charge state of interest. The phase space variables considered are $x, a, y, b, l, \delta K$, and $\delta q$. The first six variables form three canonically conjugate pairs in which the map is symplectic when there's no acceleration. The origin of the coordinate system is the position of the reference particle, which lies along the optic axis. The variable $l$ is proportional to the time variable by the expression,

$$
l=-\left(t-t_{0}\right) v_{0} \gamma /(1+\gamma)
$$

where $t_{0}$ and $v_{0}$ are the arrival time and velocity of the reference particle, respectively. To the particle of interest we assign the arrival time, $t$, and its total energy over $m_{0} c^{2}$ as $\gamma$. The fractional difference in kinetic energy relative to the reference particle is $\delta K=\left(K-K_{0}\right) / K_{0}$ and similarly for the charge state variable $\delta q$.

\section{TRANSPORT SYSTEMS}

The transport schemes are described in two sections. The discussion is mostly technical and only minor detail 
can be offered about the reasoning behind the symmetries. The reader is referred elsewhere for such discussions [8].

\section{TRANSPORT SYSTEMS}

The transport schemes are described in two sections. The discussion is mostly technical and only minor detail can be offered about the reasoning behind the symmetries. The reader is referred elsewhere for such discussions [8].

\subsection{Parallel-Shifted System}

The first system is devised for a stripped beam of $238 \mathrm{U}$ exiting a foil at $12 \mathrm{MeV} / \mathrm{u}$. The $q=75$ charge state is taken as the reference since it is to be the most populated state after the strip. Charge states 73 to 77 are transported through. The values of the emittance are $10 \pi \mathrm{mm}-\mathrm{mr}$ in the transverse plane and $5 \pi \mathrm{keV} / \mathrm{u}$ 'ns in the longitudinal.

The top portion of Figure 1 illustrates a plot with the layout of all the elements. The beam comes in from left and goes through a series of quadrupoles labeled from 1 to 12 and two pairs of dipoles located as shown. The system is mirror symmetric about the center of the superconducting cavity structure, labeled SRF. The total path length of the channel is $12.9 \mathrm{~m}$.

The map of the system from the beginning to the midsection of the SRF has some important characteristics. The path length is independent of charge state so that the first order term $(l, \delta q)$ vanishes. Furthermore, the system is not truly achromatic at this point, since the dispersion $(x, \delta K)$ vanishes but $(a, \delta K)=-0.1 \mathrm{rad}$. Although the dispersion does not vanish throughout the entire region of cavity, the separation between charge states remains small and the effects are negligible. The radius of the beam stays below $6 \mathrm{~mm}$ throughout the SRF region. The cavities rebunch the beam so that to first order the bunch exits the entire system with identical beam parameters as the ones it enters the system with. The system is an achromat and isopath at the exit.

The dispersion is large enough at the entrance of the quadrupole, $\mathrm{Q}_{4}$, to consider this section as the place to skim off beam of unwanted charge states. The dispersion is $(x, \delta K)=0.75 \mathrm{~m}$ at this location, which is enough to get a $10 \mathrm{~mm}$ separation between $q$ and $q+1$ beams that have a 3 $\mathrm{mm}$ radius. The beam envelopes in $x$ and $y$ are plotted at the lower section of Figure 1 for two different charge state combinations.

\subsection{Bending Systems}

This transport system accepts beams stripped right after the medium energy range section. The $238 \mathrm{U}$ beam comes out of the foil at about $80 \mathrm{MeV} / \mathrm{u}$ and we assume that the strip predominantly populates the $q=90$ state. The acceptance is formed for the range $q=88$ to 90 . The values of the emittance are expected at about $4 \pi \mathrm{mm}-\mathrm{mr}$ in the transverse plane and $20 \pi \mathrm{keV} / \mathrm{u}$ ns in the longitudinal.

The upper section of Figure 2 illustrates the layout of the system. The system brings the beam back around by a $180^{\circ}$ bend with a translation less than $7.5 \mathrm{~m}$. This was a technical minimum from imposing a maximum field limit of 4.5 Tesla to the dipoles. The bend feature is used to conserve space and rf power resources for the facility.

This system also has symmetry about the SRF midpoint. Unlike the previous system, this one has both $(x, \delta K)$ and $(a, \delta K)$ vanishing at the SRF and at the exit. The same principle is used to apply $(l, \delta q)=0$ at the SRF and at the exit of the system, where a small $10^{\circ}$ outward is followed by a $55^{\circ}$ bend inward in this case. We note that it is not possible to form a full achromat unless there is at least one horizontal focusing quadrupole between two dipoles; hence, the reason $(a, \delta K)$ could not vanish simultaneously with $(x, \delta K)$ in the case of the parallelshifted system. Two dipoles close to each other essentially behave as one, except for the isopath character that they add to the system by bending in the opposite direction. The rebunching allows the beam to be matched to the acceleration section that follows.

The maximum dispersion between charge states occurs at the entrance to quadrupole $\mathrm{Q}_{3}$ and is the ideal place to skim unwanted charge states away. Almost $6 \mathrm{~mm}$ between $q$ and $q+1$ exists there between beams of $\sim 4 \mathrm{~mm}$ diameter. The beam envelopes in $x$ and $y$ are plotted at the lower section of Figure 2 for two different charge state combinations.

The plots in Figure 3 illustrate the effects of the transfer map on the phase space of a beam consisting of charge states $\mathrm{q}=89,90$, and 91 . From left to right, the plots correspond to the $x-a, y-b$, and $l-\delta K$ phase space plots. The relative distortions between the different charge states are largely due in part to second order chromatic aberrations. Although there is some growth in emittance, the bulk of the occupied phase space regions remain within tolerable of the acceptance for the linac structures that are intended to proceed.

\section{CONCLUSIONS}

Progress has been made in obtaining systems for transporting multiple charge state beams. Further, studies may help to reduce the higher order effects of the transport, for example, by strategically applying multipole fields.

\section{REFERENCES}

[1] J.A. Nolen, et al., "An Advanced ISOL Facility Based on ATLAS," Proc. Of the $8^{\text {th }}$ International Conference on Heavy Ion Accelerator Technology (HIAT98), AIP Conference Proceedings 473, Ed. K. W. Shepard, p. 477, and "Report to Users", ATLAS Facility. ANLATLAS-99-1, March 1999.

[2] P.N. Ostroumov, et al., "A high-power heavy-ion driver accelerator for an advanced rare-isotope accelerator facility in the United States". ICFA Beam Dynamics Newsletter, No. 20, August 1999, p. 60. 
[3] C. Scheidenberger, Th. Stohlder, W.E. Meyerhof, H. Geissel, P.H. Mokler, B. Blank, Nucl. Inst. Meth. Phys. Res. B 142 (1998) 441-462.

[4] Atomic Data and Nuclear Data Tables 69, 217-238 (1998).

[5] M. Berz, Modern Methods in Particle Beam Optics, Academic Press, San Diego, 1999.

[6] COSY Infinity Web page and Manual. http://www.beamtheory.nscl.msu.edu/cosy <http://www.cern.ch/accelconf/templates.html $>$

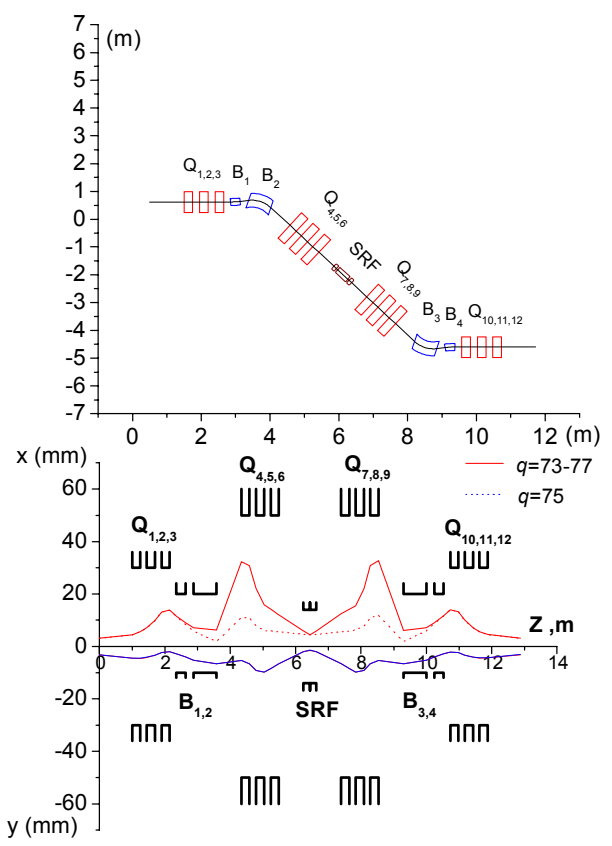

Figure 1. Parallel-shifted translation system for transporting and selecting multiple $q$ state beams at $12 \mathrm{MeV} / \mathrm{u}$ (top). Plot of envelopes (bottom).
[7] A.A. Geraci, T.A. Barlow, M. Portillo, J.A. Nolen, K.W. Shepard, K. Makino and M. Berz, Calculation of Rado-Frequency and Electrostatic Structures Using Map-Oriented Beam Optics (to be published).

[8] K.G. Steffen, "High Energy Beam Optics", John Wiley \& Sons, 1964.

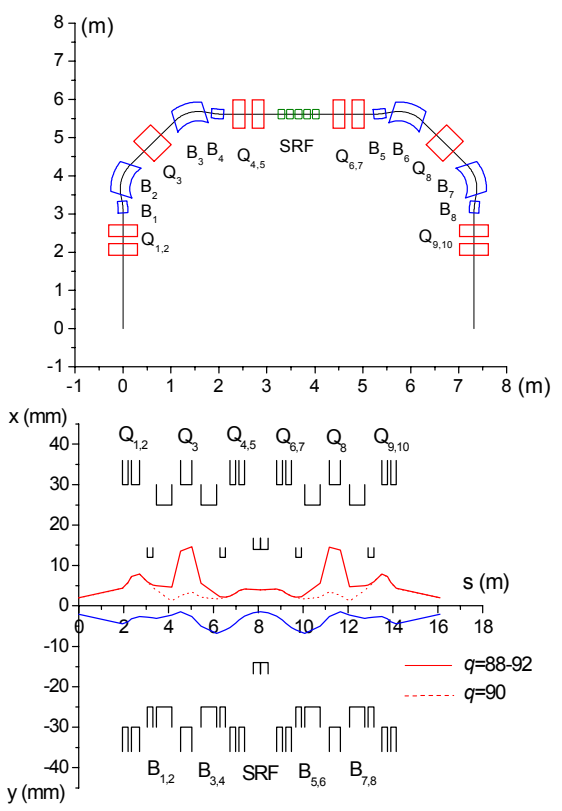

Figure 2. System for selecting multiple $q$ state beams through a $180^{\circ}$ bend at $80 \mathrm{MeV} / \mathrm{u}$ (top). Plot of enevelopes (bottom).

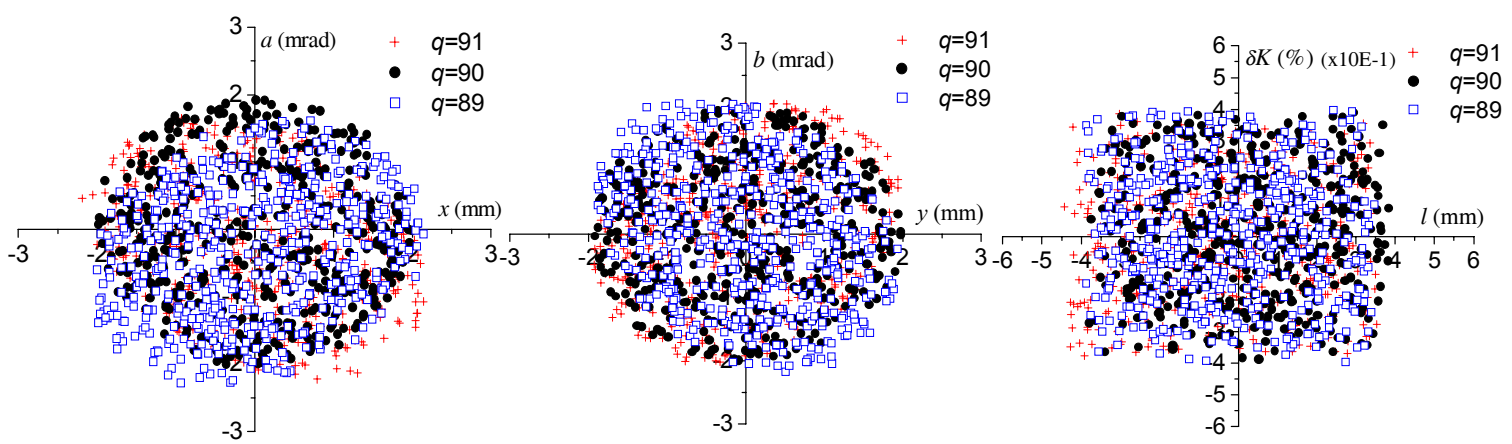

Figure 3. Plots of particles occupying the $x-a, y-b$, and $l-\delta K$ phase space at the exit of the $180^{\circ}$ bend system (left to right). The selected charge states are $q=89,90$, and 91 and the calculation was carried out to 3rd order. 